\title{
Hard X-ray observations of Extremely Red Objects
}

\author{
MARCELla BRUSA \\ Dipartimento di Astronomia, Università di Bologna, via Ranzani 1, I-40127 Bologna, Italy
}

Received date will be inserted by the editor; accepted date will be inserted by the editor

\begin{abstract}
Extremely Red Objects (EROs, R-K $>5$ ) constitute a heterogenous class of extragalactic sources including high redshift elliptical galaxies, dusty star-forming systems and heavily obscured AGNs. Hard X-ray observations provide an unique and powerful tool to uncover obscured nuclear or star-forming activity. We present the results of XMM-Newton observations of the largest sample of EROs available to date (about 450 objects over a contiguous area of $700 \operatorname{arcmin}^{2}$ ). Five of the 46 hard X-ray selected sources brighter than $3 \times 10^{-15} \mathrm{cgs}$ in the $2-10 \mathrm{keV}$ band, are associated with EROs. All of the X-ray detected EROs show rather extreme X-ray-to-optical flux ratios, suggesting the presence of highly obscured AGN activity. We also report on the X-ray stacking analysis of spectroscopically identified EROs in the Chandra Deep Field South.
\end{abstract}

Key words: galaxies: active - galaxies: starburst - surveys - X-rays: galaxies

\section{Introduction}

Extremely Red Objects (EROs, $R-K>5$ ) show the bulk of the emission in the near-infrared band and are associated with extremely faint optical counterparts that have so far limited the identification process. The first available optical-infrared spectra (e.g., Cimatti et al. 1998; Smail et al. 1999; Pierre et al. 2001) and the recent results from the K20 survey (Cimatti et al. 2002) indicate that EROs counterparts lie at high redshifts. In particular, their colors are consistent with those of early-type passively evolving galaxies, dusty starforming systems and Active Galactic Nuclei (AGN) reddened by dust and gas in the redshift range $z=0.8 \div 3$. The relative fraction of these different subclasses among the ERO population is a key parameter in the study of the galaxy evolution and can constrain models which link the formation of massive elliptical galaxies and the onset of AGN activity (Granato et al. 2001; Almaini et al. 2002).

Hard X-ray observations provide a powerful tool to uncover AGN among the EROs population. Indeed, a sizeable fraction of hard X-ray sources recently discovered in deep Chandra and XMM-Newton surveys are associated with EROs, the exact value depending on the limiting fluxes reached in the optical and X-ray bands (Brusa et al. 2002a; Mainieri et al. 2002; Alexander et al. 2002). Moreover, the excellent imaging capabilities of Chandra enable to probe the average Xray properties of EROs even beyond the limiting flux of deep surveys, using the stacking analysis technique.

Correspondence to: brusa@bo.astro.it
In this framework we have developed two complementary programs of hard X-ray observations of EROs making use of both Chandra and XMM-Newton data. First, we present the results of a XMM-Newton survey on a large, complete sample of EROs which is the most suitable one to estimate the fraction of AGN-powered EROs among the optically selected population. Then, we have constrained the high-energy properties of a sample of spectroscopically identified nonAGN EROs in the Chandra Deep Field South (CDFS) via stacking analysis.

Throughout the paper, a cosmology with $H_{0}=70 \mathrm{~km} \mathrm{~s}^{-1}$ $\mathrm{Mpc}^{-1}, \Omega_{m}=0.3$ and $\Omega_{\Lambda}=0.7$ is adopted.

\section{XMM-Newton observation of the "Daddi Field"}

\section{1. $X$-ray data analysis and results}

We have started an extensive program of multiwavelength observations of the largest sample of EROs available to date ( $\sim 450$ sources), selected in a contiguous area over a $\sim 700$ $\operatorname{arcmin}^{2}$ field (the "Daddi field", Daddi et al. 2000) and complete to a magnitude limit of $K=19.2$. Deep optical $(R \sim 26.2$ at the $3 \sigma$ level) photometry is available (see Daddi et al. 2000 for details in optical and near-infrared data reduction) and VIMOS spectroscopy is planned at VLT.

The Daddi field was observed by XMM-Newton on August 3, 2001 for a nominal exposure time of $\sim 50 \mathrm{ks}$. The XMMNewton data were processed using version 5.2 of the Science Analysis System (SAS). The event files were cleaned 


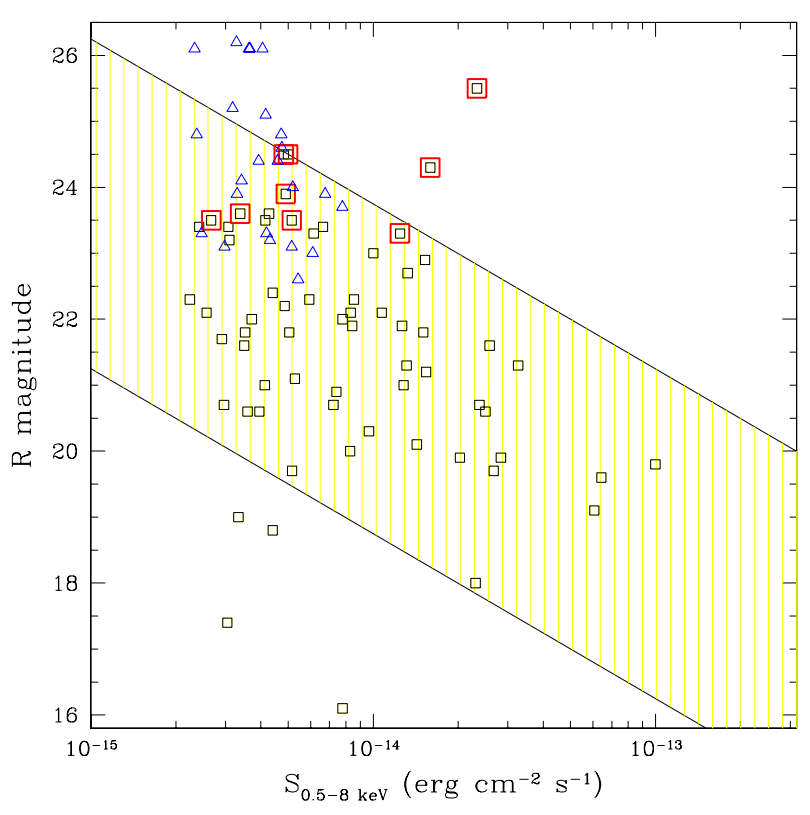

Fig. 1. R-band magnitude vs. the $0.5-8 \mathrm{keV}$ flux for the 91 sources detected in the XMM-Newton observation. Squares are sources detected both in the $\mathrm{K}$-band $(\mathrm{K}<19.2)$ and in the $\mathrm{R}$-band $(\mathrm{R}<26.2)$ images, triangles are sources detected in the R-band image only. Enlarged squares are EROs. The dashed area corresponds to the region of $0.1<\mathrm{f}_{x} / \mathrm{f}_{o}<10$.

up from hot pixels and soft proton flares; the resulting exposure time is $\sim 35 \mathrm{ks}$ in the MOS1 and MOS2 detectors and $\sim 30 \mathrm{ks}$ in the $p n$ detector. The EBOXDETECT task, the standard $S A S$ sliding box cell detect algorithm, was run on the $0.5-2,2-8$ and $0.5-8 \mathrm{keV}$ cleaned events. We limited the X-ray analysis in a region of ten arcmin radius from the centre of the XMM-Newton pointing, to take into account the broadening of the XMM-Newton PSF at increasing distance from the aim point. Ninety-one sources were detected in the full X-ray band with a threshold in Poisson probability of $5 \times 10^{-6}$; the corrisponding flux limit is $\sim 3 \times 10^{-15} \mathrm{erg}$ $\mathrm{cm}^{-2} \mathrm{~s}^{-1}$ assuming a power-law spectrum with $\Gamma=1.8$ and Galactic absorption $\left(\mathrm{N}_{H}=5 \times 10^{20} \mathrm{~cm}^{-2}\right)$.

The X-ray centroids have been corrected for systematic errors with respect to the optical positions of three bright quasars in the field (Hall et al. 2000) and then were cross-correlated with the $K$-band and $R$-band catalogs. We searched for near-infrared counterparts within a radius of $6^{\prime \prime}$ from the X-ray position; we note, however, that the $75 \%$ of the sources lie within a $3^{\prime \prime}$ radius circle. The results and the optical identifications are summarized in Fig. 1: $80 \%$ of the $0.5-8 \mathrm{keV}$ sources have at least one near-infrared counterpart down to the $\mathrm{K}$-band magnitude limit; four sources are not detected at the R-band limit. Nine out of ninety-one sources are associated with EROs ( $\sim 10 \%)$; the fraction is the same (5/46) for the 2-8 keV sample. We refer to Brusa et al. (2002a) for a more detailed discussion on the XMM-Newton data analysis, the associations of X-ray sources with optical counterparts and the analysis of $\mathrm{X}$-ray colors.

\subsection{AGN fraction in optically selected samples}

The AGN fraction among optically selected EROs samples is still very uncertain (Brusa et al. 2002a; Mainieri et al. 2002), and strongly dependent on the limiting fluxes reached in both the X-ray and near-infrared bands. About 370 EROs fall within the XMM-Newton area analysed in this work. The fraction of AGN-powered, hard X-ray selected EROs at the near-infrared $(\mathrm{K}=19.2)$ and $\mathrm{X}$-ray flux limits of our survey is therefore $\sim 1 \div 2 \%(5 / 370)$. This fraction rises up to $14 \%$ at at $\mathrm{K} \lesssim 20.1$ and $\mathrm{S}_{2-10} \gtrsim 4 \times 10^{-16} \mathrm{erg} \mathrm{cm}^{-2} \mathrm{~s}^{-1}$ limiting fluxes of the CDFN observation (Alexander et al. 2002), supporting the idea that the bulk of the EROs population is not related to active phenomena.

It is worth noticing that these AGN-powered EROs, as well as others examples of hard $\mathrm{X}$-ray selected EROs show a relatively well-defined correlation between the $\mathrm{X}$-ray fluxes and the optical magnitude around $\mathrm{f}_{\mathrm{X}} / \mathrm{f}_{\mathrm{O}} \simeq 10$. This correlation is shifted by one order of magnitude from the one found by ROSAT for soft X-ray selected quasars (Hasinger et al. 1998) and recently extended by Chandra and XMM-Newton observations to hard X-ray selected sources (Alexander et al. 2002; Lehmann et al. 2001). The most plausible explanation of such a high X-ray-to-optical flux ratio in these objects is the presence of high obscuration towards the active nuclear source. This hypothesis is strongly supported by optical identifications available for a handful of objects (Cowie et al. 2001; Mainieri et al. 2002; Hornschemeier et al. 2001) and by the $\mathrm{X}$-ray spectral analysis (see Sect. 4).

\section{Stacking EROs in the CDFS}

The K20 EROs sample (Cimatti et al. 2002) in the Chandra Deep Field South (CDFS) area includes 48 objects at $K_{s}<20$. For the purposes of the present work we consider only the 21 spectroscopically identified EROs which have been classified in two categories: 13 "dusty", i.e. objects showing at least an emission line (tipically [OII] $\lambda 3727$ ) over a red continuum, and 8 "old", i.e. objects with an absorptionline spectrum, consistent with that of early-type, passively evolving galaxies, distributed on a similar range of redshifts $(z=0.8 \div 1.6)$.

For the X-ray analysis, we made use of the public CDFS one megasecond Chandra observation available in the archive (Giacconi et al. 2002). At first, we have searched for individual X-ray emission from all the objects in the EROs spectroscopic redshift catalog of Cimatti et al. (2002). Only one source, a dusty ERO at $z=1.327$, was individually detected in the one megasecond exposure. The optical position is almost coincident $(\Delta<0.3$ ", within the accuracy of the K-band position) with the Chandra source CXO CDFS J033213.9-274526 in the Giacconi et al. (2002) cata$\log$. The very hard $\mathrm{X}$-ray spectrum and the high intrinsic Xray luminosity unambiguously reveal the presence of an obscured AGN in this source. Indeed, this object is undetected in the soft band, implying an intrinsic column density larger than $4 \times 10^{23} \mathrm{~cm}^{-2}$, and an unabsorbed hard X-ray luminosity $\mathrm{L}_{2-10}>4 \times 10^{43} \mathrm{erg} \mathrm{s}^{-1}$; its properties are then more 

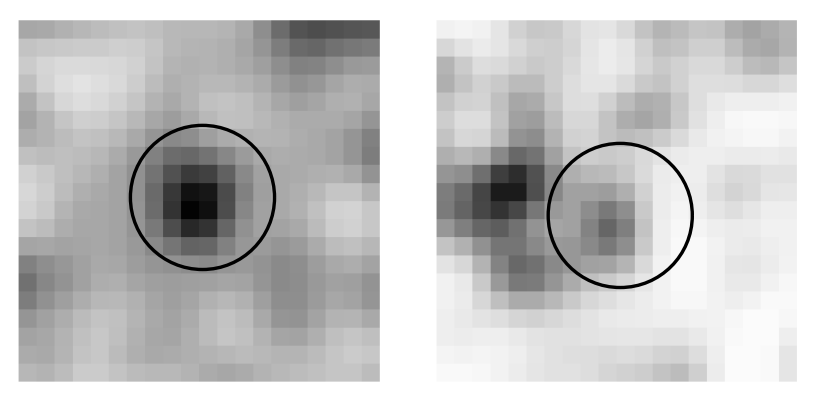

Fig. 2. Stacked full-band images of the "dusty" (left panel) and "old" (right panel) EROs. The images are 9x9 arcsec and have been smoothed with a gaussian of $\sigma=1.5$ pixel (approx $0.7^{\prime \prime}$ ). The circles are centered on the stacking position and have a radius of $2^{\prime \prime}$. The detection significance of the summed counts is $4.2 \sigma$ for the "dusty" and $<2 \sigma$ for the "old". The bright spot in the right panel at about 4 " from the stacked position, corresponds to an individually detected object.

extreme than those inferred from the $2 \mathrm{Ms}$ CDF-N spectral analysis (Vignali et al. 2002). Given the small area sampled and the spectroscopic incompleteness, the present ERO sample is not suitable for a reliable estimate of the AGN fraction among the EROs population. However, the present low detection rate of AGN among EROs (1/21) confirms that the bulk of the EROs population is not related to active phenomena (see Sect. 2.2).

In order to constrain the average $\mathrm{X}$-ray properties of individually undetected EROs, we have applied the stacking technique (Brandt et al. 2001; Nandra et al. 2002) separately for the two classes of objects. The samples consist of 12 "dusty" and 8 "passive" EROs, with average redshifts of $z=1.053$ and $z=1.145$, respectively. We performed the detection in the three standard X-ray bands $(0.5-8,0.5-2$ and 2-8 keV) and in the $1-5 \mathrm{keV}$ band which roughly corresponds to the rest-frame hard X-ray band at the average redshift of the samples. We refer to Brusa et al. (2002b) for details on the $\mathrm{X}$-ray data analysis.

In Fig. 2 we show the summed images in the full $(0.5-8 \mathrm{keV})$ band. The most important finding is that the two spectroscopically classes of EROs have different X-ray properties.

\subsection{An X-ray dichotomy}

The X-ray emission from "old" EROs remains undetected in all the considered energy bands, for a total effective exposure time of $\sim 7.0 \mathrm{Ms}$. Assuming a thermal emission model with $\mathrm{kT}=1 \mathrm{keV}$ and solar metallicity, fully consistent with the average X-ray spectrum of nearby elliptical galaxies (e.g., Pellegrini 1999), the $3 \sigma$ upper limit on the $0.5-2 \mathrm{keV}$ luminosity is $L_{X}<10^{41} \mathrm{erg} \mathrm{s}^{-1}$.

An excess of counts above the expected background level is clearly present in the "dusty" sample, for a total effective exposure time of $10 \mathrm{Ms}$. The signal is stronger in the full band (99.997\% confidence level assuming a Poisson distribution, corresponding to about $4.2 \sigma$ ) and it is still present, although at a slightly lower confidence level, in the soft and 1-5 keV band, while it is not statistically significant in the hard band. Assuming a $\Gamma=2$ power-law spectrum plus Galactic absorption $\left(\mathrm{N}_{H}=8 \times 10^{19} \mathrm{~cm}^{-2}\right)$, the $3 \sigma$ upper limit on the average band ratio $(\mathrm{H} / \mathrm{S}<0.85$, where $\mathrm{H}$ and $\mathrm{S}$ indicates the hard band and soft band counts, respectively), corresponds to an absorption column density lower than $10^{22} \mathrm{~cm}^{-2}$ (see Fig. 4 in Alexander et al. 2002). Given that the hard X-ray spectrum of nearby starburst galaxies is well-represented by powerlaw models with slopes in the range $1.8 \div 2.5$ and low intrinsic absorption (e.g., Ptak et al. 1999; Ranalli et al. 2002), we adopted $\Gamma=2.1$ to compute $X$-ray fluxes and luminosities. With this assumption, the stacked count rate in the $1-5 \mathrm{keV}$ band corresponds to an average $2-10 \mathrm{keV}$ rest-frame luminosity of $\sim 8 \times 10^{40} \mathrm{erg} \mathrm{s}^{-1}$, at the mean redshift $z=1.053$. The inferred, average $\mathrm{X}$-ray luminosity is around one order of magnitude larger than that of normal spiral galaxies (Matsumoto et al. 1997), it is similar to that of the starburst galaxy M82 (e.g., Griffiths et al. 2000; Kaaret et al. 2001) and about 3 times lower than that of NGC 3256, one of the most X-ray luminous starburst in the local Universe (e.g., Moran, Lehnert, \& Helfand 1999).

The starburst scenario is supported also by the optical data. Indeed, following Cimatti et al. (2002), we recomputed the average spectrum of the 12 EROs we used in the X-ray stacking analysis. The average optical spectrum shows a very red and smooth continuum, with a strong [OII] $\lambda 3727$ emission and lacks of the presence of clear AGN indicators such as $[\mathrm{NeV}] \lambda 3426$, suggesting that the optical emission is dominated by star-forming systems.

\section{Hard X-ray properties of EROs}

We have collected from the literature a sample of hard Xray selected EROs serendipitously detected in moderate-deep and deep Chandra and XMM-Newton observations (Hornschemeier et al. 2001; Alexander et al. 2002; Mainieri et al. 2002). From the observed $X$-ray band ratio, we have computed for each ERO the corresponding X-ray column density for a source with a typical AGN power-law spectrum $(\Gamma=1.8)$ at $z=1$, while for the sources in the Lockman Hole we adopted the best-fit value quoted by Mainieri et al (2002) and corrected to $z=1$ for unidentified sources. The results are reported in Fig. 3. Almost all of the individually detected sources are consistent with intrinsic column densities in excess of $10^{22} \mathrm{~cm}^{-2}$. The so far individually detected EROs have high $\mathrm{X}$-ray luminosity $\left(\mathrm{L}_{X}>10^{43} \mathrm{erg} \mathrm{s}^{-1}\right.$, in a few case even larger than $10^{44} \mathrm{erg} \mathrm{s}^{-1}$ ) and they actually are heavily obscured AGN, as inferred from X-ray spectral analysis (Cowie et al. 2001; Gandhi et al. 2002; Mainieri et al. 2002). Therefore, hard X-ray selected EROs (or at least a fraction of them) have properties similar to those of Quasars 2, the highluminosity, high redshift type II AGNs predicted in X-Ray Background synthesis models (e.g. Comastri et al. 1995).

Stacking analysis of those objects not individually detected in the X-rays suggests a softer X-ray spectrum than that of AGN-powered EROs (see also Alexander et al. 2002). More- 


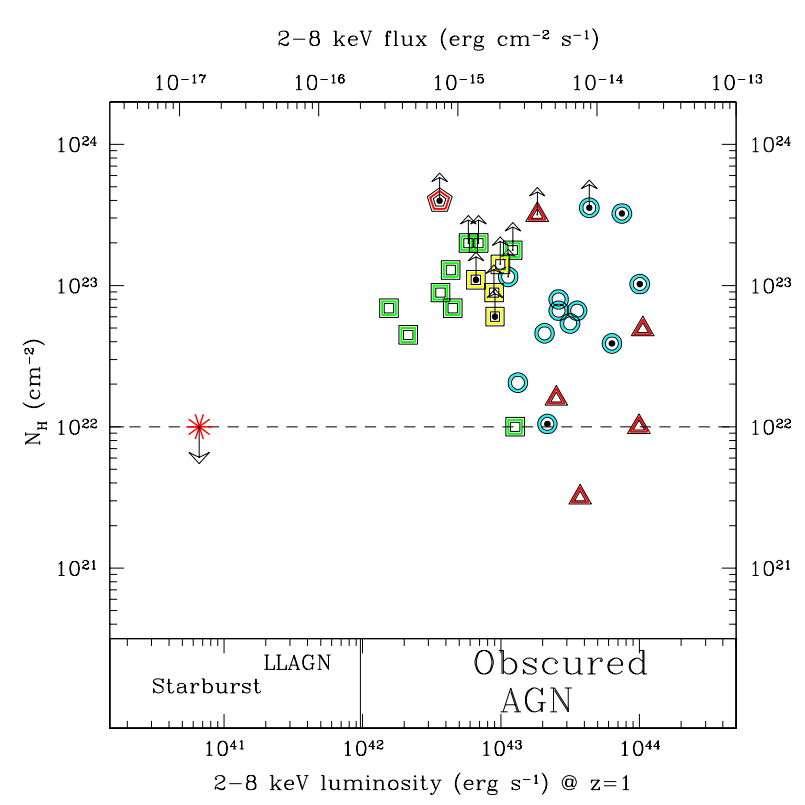

Fig. 3. X-ray absorption column density vs. hard X-ray flux for a sample of AGN-powered EROs, serendipitously detected in hard $\mathrm{X}$-ray surveys. Different symbols refer to different samples as follows: triangles = "Daddi" field; squares $=$ CDFN (Hornschemeier et al. 2001; Alexander et al. 2002); circles = Lockman Hole (Mainieri et al. 2002). The individually detected AGN in the K20 CDFS area is marked with a pentagon; the result from the stacking analysis of the "dusty" sample is marked with an asterisk. Dot-filled symbols refer to sources with measured redshift.

over, the results from the CDFS K20 EROs sample suggest that different spectroscopic classes of EROs are characterized by different high-energies properties. Indeed, "dusty" EROs are moderately luminous hard X-ray emitters and their average $\mathrm{X}$-ray spectrum $\left(\mathrm{N}_{H}<10^{22} \mathrm{~cm}^{-2}\right)$ is consistent with that measured for nearby star-forming galaxies (e.g. Dahlem, Weaver \& Heckman 1998). Early-type galaxies among EROs are not detected in the $\mathrm{X}$-rays and their $3 \sigma$ upper limit on the soft $\mathrm{X}$-ray luminosity is consistent with the emission from nearby elliptical galaxies (Pellegrini 1999).

\section{Conclusions}

The most important results can be summarized as follows:

- Hard X-ray observations turned out to be extremely useful to pick-up AGN-powered EROs in optically selected samples. The fraction of AGN among EROs is in the range $1 \div 15 \%$, depending on the limiting fluxes in the $\mathrm{X}$-ray and near-infrared bands.

- AGN-powered EROs are X-ray luminous and obscured objects. The intrinsic column densities inferred for a sample of hard X-ray selected EROs are in excess of $10^{22}$ $\mathrm{cm}^{-2}$.

- "Dusty" EROs are relatively unobscured $\left(\mathrm{N}_{H}<10^{22}\right.$ $\mathrm{cm}^{-2}$ ) hard X-ray sources and their X-ray properties are consistent with those of nearby star-forming galaxies.

- "Old" EROs are not detected in the X-rays.
Acknowledgements. I thank A. Comastri, A. Cimatti, E. Daddi, M. Mignoli, L. Pozzetti and C. Vignali for fruitful discussion and for a careful reading of the manuscript. This work was supported by ASI I/R/113/01 and MIUR Cofin-00-02-36 grants.

\section{References}

Alexander D.M., Vignali C., Bauer F.E., et al.: 2002, Astron. J. 123, 1149

Almaini O., et al.: 2002, Mon. Not. R. Astron. Soc. in press (astro$\mathrm{ph} / 0108400)$

Brandt, W.N., Hornschemeier, A.E., Schneider, D.P., et al.: 2001, Astrophys. J. 558, L5

Brusa, M., Comastri, A., Daddi, E., Cimatti, A., Vignali, C.: 2002a, MPE Report 279, 267

Brusa, M., Comastri, A., Daddi, E., Cimatti, A., Mignoli, M., Pozzetti, L.:2002b, submitted to Astrophys. J. Lett.

Cimatti, A., Andreani, P., Rottgering, H., Tilanus, R.: 1998, Nat. 392, 895

Cimatti, A., et al.: 2002, Astron. \& Astroph. 381, L68

Comastri, A., Setti, G., Zamorani, G., Hasingher, G.:1995, Astron. \& Astroph. 296, 1

Cowie ,L.L., et al.: 2001, Astrophys. J. 551, L9

Crawford, C.S., Fabian, A.C., Gandhi, P., Wilman, R.J., Johnstone, R.M.: 2001, Mon. Not. R. Astron. Soc. 324, 427

Daddi E., Cimatti, A., Pozzetti L., et al.: 2000, Astron. \& Astroph. 361,535

Dahlem, M., Weaver, K.A., Heckman, T.M.: 1998, Astrophys. J. Suppl. 118, 401

Freeman, P.E., Kashyap, V., Rosner, R., Lamb, D.: 2002, Astrophys. J. Suppl. 138, 185

Gandhi, P., et al.: 2002, Proc. XXI Moriond Astrophysics Meeting, "Galaxy Clusters and the High Redshift Universe Observed in $X$-rays" (astro-ph/0106139)

Giacconi, R., et al.: 2002, Astrophys. J. Suppl. 139, 369

Granato, G.L., Silva, L., Monaco, P., et al.: 2001, Mon. Not. R. Astron. Soc. 324, 757

Griffiths, R.G., et al.: 2000, Science 290, 1325

Hall, P.B., et al.: 2000, Astron. J. 120, 2220

Hasinger, G., et al.: 1998, Astron. \& Astroph. 329, 482

Hornschemeier, A.E., et al.: 2001, Astrophys. J. 554, 742

Kaaret, P., Prestwich, A.H., Zezas, A., et al.: 2001, Mon. Not. R. Astron. Soc. 321, L29

Lehmann, I., Hasinger, G., Murray, S.S. \& Schmidt, M.: 2001, in "X-rays at Sharp Focus Chandra Science Symposium" (astro$\mathrm{ph} / 0109172)$

Mainieri, V., Bergeron, J., Hasinger, G., et al.: 2002, Astron. \& Astroph. 393, 425

Matsumoto, H., Koyama, K., Awaki, H., Tsuru, T., Loewenstein, M., Matsushita, K.: 1997, Astrophys. J. 482, 133

Moran, E.C., Lehnert, M.D., Helfand, D.J.: 1999, Astrophys. J. 526, 649

Nandra, K., Mushotzky, R.F., Arnaud, K., et al.: 2002, Astrophys. J. 576, 625

Pellegrini, S.: 1999, Astron. \& Astroph. 351, 487

Pierre, M., et al. 2001, Astron. \& Astroph. , 372, L45

Ptak, A., Serlemitsos, P., Yaqoob, T., Mushotzky, R.F.: 1999, Astrophys. J. Suppl. 120, 179

Ranalli, P., Comastri, A., Setti, G.C.:2002, Astron. \& Astroph. submitted

Smail, I., Ivison, R.J., Kneib, J.P., et al.: 1999, Mon. Not. R. Astron. Soc. 308, 1061

Vignali, C., Alexander, D.M., Bauer, F.E., et al.: 2002, proceedings of the 5th Italian AGN Meeting "Inflows, Outflows and Reprocessing around black holes", astro-ph/0209415 